\title{
La climatología histórica en Latinoamérica. Desafíos y perspectivas
}

La climatologie historique en Amérique latine. Défis et perspectives

Historical climatology in Latin America. Challenges and Perspectives

María del Rosario Prieto, Facundo Rojas y Leonardo Castillo

\section{(2) OpenEdition}

Journals

Edición electrónica

URL: http://journals.openedition.org/bifea/9706

DOI: $10.4000 /$ bifea.9706

ISSN: 2076-5827

Editor

Institut Français d'Études Andines

Edición impresa

Fecha de publicación: 1 agosto 2018

Paginación: 141-167

ISSN: 0303-7495

Referencia electrónica

María del Rosario Prieto, Facundo Rojas y Leonardo Castillo, « La climatología histórica en

Latinoamérica. Desafíos y perspectivas ", Bulletin de l'Institut français d'études andines [En línea], 47

(2) | 2018, Publicado el 08 agosto 2018, consultado el 04 noviembre 2020. URL : http:// journals.openedition.org/bifea/9706 ; DOI : https://doi.org/10.4000/bifea.9706

\section{(c) $(1)$}

Les contenus du Bulletin de l'Institut français d'études andines sont mis à disposition selon les termes de la licence Creative Commons Attribution - Pas d'Utilisation Commerciale - Pas de Modification 4.0 International. 


\title{
La climatología histórica en Latinoamérica. Desafíos y perspectivas
}

\author{
María del Rosario Prieto* \\ Facundo Rojas ${ }^{* *}$ \\ Leonardo Castillo***
}

\begin{abstract}
Resumen
El objetivo del presente artículo es describir y analizar el desarrollo de la climatología histórica en los países latinoamericanos, identificando las principales perspectivas que esta disciplina presenta en la región. Partimos de la hipótesis de que este campo de estudio, muy vinculado tanto a la climatología como a la historia ambiental, ha tenido abordajes muy heterogéneos, según el país y la perspectiva teórico-metodológica que se consideren, lo que podría haber dificultado su difusión y la consolidación de un campo de estudio. Sin embargo, una valiosa riqueza y potencialidad se deriva de la mencionada heterogeneidad de los estudios latinoamericanos sobre el clima. Podría tratarse de rasgos que expresen una identidad académica mestiza, donde conviven y se mixturan perspectivas que originalmente se idearon para ser trabajadas de forma aislada.
\end{abstract}

Palabras clave: climatología histórica, Latinoamérica, estado del arte, variabilidad climática, hidroclimatología

* Investigadora principal del Consejo Nacional de Investigaciones Científicas y Técnicas (CONICET), Instituto Argentino de Nivología, Glaciología y Ciencias Ambientales - Grupo de Historia ambiental (Av. Ruiz Leal s/n, Parque General San Martín, Mendoza Capital [CC 330, CP 5500]), Argentina. E-mail: mrprieto@mendoza-conicet.gob.ar

** Investigador asistente del Consejo Nacional de Investigaciones Científicas y Técnicas (CONICET), Instituto Argentino de Nivología, Glaciología y Ciencias Ambientales - Grupo de Historia ambiental, Universidad Nacional de Cuyo. E-mail: frojas@mendoza-conicet.gob.ar

*** Becario doctoral del Consejo Nacional de Investigaciones Científicas y Técnicas (CONICET), Instituto Argentino de Nivología, Glaciología y Ciencias Ambientales - Grupo de Historia ambiental, Universidad Nacional de Cuyo. E-mail: Icastillo@mendoza-conicet.gob.ar 


\title{
La climatologie historique en Amérique latine. Défis et perspectives
}

\section{Résumé}

L'objectif de cet article est de décrire et analyser le développement de la climatologie historique dans les pays latino-américains, en identifiant les principales perspectives que cette discipline présente dans la région. L'hypothèse de départ est que ce champ d'étude, très lié tant à la climatologie qu'à I'histoire environnementale, a connu des approches très hétérogènes, selon les pays et les démarches théoriques et méthodologiques, ce qui pourrait en avoir entravé sa diffusion et la consolidation d'un domaine d'étude. Cependant, I'hétérogénéité des études latino-américaines sur le climat constitue une richesse et un potentiel précieux. Il peut s'agir de traits exprimant une identité académique métisse, où des perspectives conçues à l'origine pour être travaillées de manière isolée coexistent et peuvent se combiner.

Mots-clés : climatologie historique, Amérique latine, état de l'art, variabilité climatique, hydroclimatologie

\section{Historical climatology in Latin America. Challenges and Perspectives}

\begin{abstract}
The aim of this paper is to describe and analyze the development of historical climatology in Latin America in an attempt to identify the main perspectives presented by this discipline in the region. It is based on the assumption that this field of study is mainly linked to the discussions of climatology and environmental history. Climate history has been approached in a heterogeneous way in accord with the academic tradition of the country and the theoretical-methodological perspective considered. Nevertheless, there is value in the heterogeneity of Latin American studies of climate. It includes features that express a mestizo academic identity. The varied perspectives created werebased on the idea that each one works by itself coexisting and mixing inside this identity.
\end{abstract}

Keywords: historical climatology, Latin America, state of the art, climate variability, hydroclimatology

\section{Introducción}

La avenida que se esperimentó en el presente año fué tan copiosa i abundante que no se ha visto otra mayor desde la fundación de la capital.

«Carta del Cabildo de Santiago de Chile al rey de España, 20 de diciembre de 1783» (Vicuña Mackenna,1970: 97).

La cita que encabeza este artículo muestra que las sociedades del pasado transmitían la ocurrencia de catástrofes naturales (grandes lluvias y las subsecuentes inundaciones). En muchos casos coincidían en la singularidad de la anomalía 
y su calidad de suceso nunca antes experimentado. Este tipo de aseveraciones era más común en los siglos en los que se carecía de instrumentos para medir tanto el tiempo como el caudal de los ríos. La necesidad de conocer el clima del pasado con la mayor precisión posible condujo a la Historia (en sintonía con varias disciplinas que estudian el pasado de la Tierra) a desarrollar metodologías que permitieran transformar esta información empírica, facilitada por los documentos de archivo, en datos confiables y cuantificables. Esto permitiría la elaboración de series climáticas lo más extensas posibles para, entre otras tareas, verificar la repetición de eventos catastróficos a lo largo del tiempo. La perspectiva de un cambio climático a nivel global ha incentivado estos estudios, los cuales, junto con otras disciplinas paleoclimáticas, han conducido a un mejor conocimiento del clima del pasado.

La especialidad que abarca este estudio, utilizando fuentes documentales, es la climatología histórica. En general, esta puede ser analizada desde dos perspectivas:

1. La primera consiste en la construcción de series climáticas que permiten estudiar la variabilidad climática a lo largo de los últimos siglos para obtener datos directos e indirectos sobre el clima del pasado. Su objetivo está enfocado en la búsqueda, recopilación, procesamiento e interpretación de la información sobre el clima y el tiempo anteriores a su registro (segunda mitad del siglo XIX) mediante instrumentos, depositada en archivos documentales. El proceso finaliza con la construcción de índices climáticos que permiten reconstruir, de la manera más objetiva posible, el clima de los últimos siglos. La climatología histórica se diferencia, metodológicamente, de la climatología propiamente dicha — cuya principal fuente de información se obtiene de las mediciones instrumentales - y de la paleoclimatología — que analiza los parámetros climáticos a partir de indicadores naturales, como los anillos de los árboles, los sedimentos, el polen, etc.- (Wanner et al., 2008).

2. La segunda, complementaria a la primera, estudia el impacto de las variaciones y de los cambios climáticos sobre la vida económica y social de los grupos humanos a través del tiempo. Algunos enunciados están enfocados en la vulnerabilidad de las sociedades ante las catástrofes de la naturaleza y en las distintas respuestas culturales desarrolladas por estas para adaptarse y sobreponerse a dichos riesgos naturales. Asimismo, Pfister considera como uno de los objetivos de esta disciplina la reflexión sobre los «discursos del pasado y las representaciones sociales relacionadas con el clima» (Pfister et al., 2001: 248). Desde esta perspectiva se puede ubicar a la climatología histórica en la «interfase entre la climatología y la historia ambiental» (Pfister et al., 2001: 248; Brázdil et al., 2005: 365).

Respecto a esta última temática, en Latinoamérica existe una gran cantidad de trabajos acerca de los procesos históricos que incluyen contenidos sobre el clima y que buscan respuestas a preguntas propias de las Ciencias Sociales. Algunos de estos también se mencionarán en este artículo, aunque no se ha podido ser exhaustivo en esta rama de los estudios y algunos autores no los entienden siempre dentro de los campos de la climatología histórica o de la historia ambiental. 
Según lo que ya se conoce de estos territorios, se debe mencionar que si bien existen mediciones del tiempo desde el siglo XVIII (especialmente de temperatura y presión atmosférica), sobre todo en el continente europeo, es en el último cuarto del siglo XIX cuando en Latinoamérica se crean las primeras oficinas meteorológicas y comienza un registro sistematizado de los fenómenos meteorológicos. Es importante señalar, además, la labor pionera de los aficionados a la Meteorología. Esta ciencia contribuyó con su esfuerzo a un mayor conocimiento del clima previo a la sistematización de la información climática.

La climatología histórica se ha desarrollado en Europa con una ventaja considerable en tiempo y dimensión con respecto a Latinoamérica. Un texto precursor en este trayecto es el de Le Roy Ladurie (1990 [1967]), que constituyó un hito en su obra y una referencia para este tipo de estudios.

Desde finales de la década de 1960 se han multiplicado las investigaciones que tienen como objetivo no solo la reconstrucción del clima, sino también el estudio y la comprensión de la vulnerabilidad de las economías y de las sociedades del pasado (Pfister et al., 2001). Este es el caso de la tesis de Florescano (1969), la cual constituye a nuestro entender el primer estudio basado en fuentes documentales que interpreta las relaciones entre el clima y la sociedad mexicana desde estándares científico-académicos contemporáneos. Algunos años más tarde, Prieto (1981; 1983; 1985) realizó un estudio similar para el caso de Mendoza-Argentina. En primer lugar, estudió las fuentes históricas necesarias para realizar una historia del clima regional; posteriormente, determinó las variaciones climáticas (de temperatura y precipitaciones) ocurridas durante la Colonia; y, por último, examinó los esfuerzos de los españoles por sobrevivir en un clima tan riguroso como el de aquellas áridas regiones.

Entre las investigaciones enfocadas en la relación clima-sociedad destacamos los trabajos de Cushman. En su artículo «Humboldtian Science, Creole Meteorology, and the Discovery of Human-Caused Climate Change in South America» analiza la obra de Humboldt. Este autor alemán, que trabajó en la cuenca del Lago Valencia en Venezuela y en la costa árida del Perú, considera que las actividades humanas protagonizadas por los conquistadores españoles fueron la causa de cambios climáticos de gran escala ocurridos en América Latina. Esto habría influido no solo en sus discípulos contemporáneos, sino también en el pensamiento ambiental actual (Cushman, 2011).

El objetivo del presente artículo es analizar el desarrollo de la climatología histórica y su producción en Latinoamérica y el Caribe1, considerando las principales perspectivas y discusiones que han surgido en este campo de estudio.

1 Si bien parte del Caribe fue colonizado por Francia e Inglaterra, y en menor medida por Holanda, durante varios siglos ha existido un claro predominio de España y Portugal con relación a la conquista y la colonización de ese vasto territorio, que se extiende al sur de EE. UU. 


\section{SOBRE LAS FUENTES DOCUMENTALES}

La fuerte presencia española en América incidió en la naturaleza y en la extensión temporal de las fuentes utilizadas para realizar estudios de climatología histórica en la región (Prieto \& García-Herrera, 2009). En cuanto a su naturaleza, estas fuentes provienen de documentos en su mayoría escritos por españoles tanto en España como en América y se puede obtener, a partir de ellos, información acerca de la idiosincrasia de quienes los produjeron. En cuanto a su amplitud en el tiempo, este tipo de fuentes pueden extenderse no más allá de los albores del siglo XVI. En esos momentos se produjeron los primeros documentos que daban cuenta de las novedades sobre las Indias, remitidas a la península ibérica por los recién llegados. Por su parte, las fuentes documentales provenientes del proceso de conquista y colonización portuguesa en América han sido más escasas y discontinuas².

Entre los archivos españoles se pueden citar de manera especial las Actas Capitulares, elaboradas en función de las reuniones semanales de los integrantes de los cabildos de las ciudades americanas (entre principios del siglo XVI y principios del siglo XIX). Asimismo, es fundamental — junto con las importantes colecciones de documentos conservados en los numerosos archivos nacionales, provinciales y municipales de la región - el acervo depositado en el Archivo General de Indias (AGI) en Sevilla (García-Herrera et al., 2003). Aquí se concentra toda la documentación enviada y recibida desde y hacia América hasta el siglo XIX, en particular la correspondencia que contenía noticias sobre eventos que afectaban la economía regional, como las sequías, las inundaciones o las abundantes precipitaciones. También se deben incluir los mapas históricos usados en la región para reconstruir tanto la historia de determinados ambientes como para verificar los cambios de curso de los grandes ríos y sus efectos sobre las incipientes ciudades americanas. Existen, además, significativos informes que se extienden desde 1785 hasta 1805 y tratan acerca de eventos climáticos, tales como las «Relaciones sextrimestrales de aguas, cosechas y otros particulares» de fines del siglo XVIII conservados en el AGI. Estos informes proveen de datos directos sobre el clima y la agricultura de México, Argentina, Bolivia, Chile, Paraguay, Cuba, Puerto Rico y Guatemala. En el período colonial, el origen común de la información les confería homogeneidad a estas fuentes. Sin embargo, en las décadas siguientes, durante los primeros momentos de los Estados republicanos predominaron el desorden y la heterogeneidad en las modalidades para obtener y clasificar la información. Recién hacia la segunda mitad del siglo XIX se moderniza la manera de redactar la información administrativa y se incorporan, progresivamente, nuevas fuentes que detallan eventos climáticos, como es el caso de los periódicos. En esta línea se pueden incluir estudios que utilizan la información de fuentes documentales de origen español, ya sea de tipo gubernamental o eclesiástico

2 Da Guia Santos Gareis afirma que «el análisis de los datos se basa en la información obtenida a partir de la literatura existente, debido a la escasez e incluso inexistencia de documentación primaria sobre el tema en los archivos brasileños» (Da Guia Santos Gareis et al., 1997: 103). 
(rogativas, diezmos, tributos, alcabalas, actas de nacimientos y defunciones, entre otros), como indicadores de eventos y de cambios climáticos (sequías, granizadas, heladas, huracanes, epizootopías, plagas, etc.). Para la región Piura, en el Perú, Schlüpmann (2003) analiza, por ejemplo, la utilidad del diezmo como medida de producción agraria y, por extensión, de eventos climáticos. Para la provincia de Chiapas, reino de Guatemala, en el período colonial, Arrioja Díaz Virruell (2016) ensaya una perspectiva que no se limita solo a examinar la variabilidad del clima y la presencia de los fenómenos naturales en sí mismos, sino que además analiza los contextos en los que surgen y evolucionan con la intención de evidenciar que todo «desastre» o «catástrofe» natural es el resultado de una confluencia de elementos naturales, socioeconómicos y políticos.

Cada país o región latinoamericana tiene sus rasgos geográficos específicos y, en consecuencia, sus propias características climáticas que pueden oscilar entre sequedad extrema y niveles de humedad muy significativos. Para los habitantes de Latinoamérica se comprueba que estos eventos extremos del clima tenían mayor importancia que los períodos normales con relación a su supervivencia. Es por ello que, en general y a diferencia de Europa, se cuenta con mayor información sobre la escasez o abundancia de agua que sobre la temperatura. Esto, debido a que Latinoamérica está en la zona intertropical que se caracteriza por una variación menos importante de las temperaturas que la zona templada. En la etapa de dominio español es muy difícil determinar esta última con anterioridad al siglo XVIII, salvo en zonas templadas con cultivos como la vid o el trigo, los cuales permiten aplicar el método fenológico (por ejemplo, retraso o adelanto de las cosechas) debido a las heladas tempranas o tardías (Prieto, 1983).

Para fines del siglo XVIII y principios del siglo XIX ya se cuenta, en algunos países, con fragmentos de series de temperatura obtenidas por aficionados y estudiosos del clima, además de los informes meteorológicos de las expediciones científicas españolas que dejaron su simiente en las incipientes ciudades latinoamericanas.

En síntesis, y de acuerdo con la definición brindada líneas atrás, veremos que en cada territorio de esta amplia región han prevalecido ciertos temas respecto de algunos otros. Esto, de acuerdo no solo con la realidad climática que les tocó vivir, sino también con los intereses y las valoraciones de los grupos sociales que recopilaban la información. Por la abundancia de trabajos destacan dos países en este contexto: México y Argentina.

\section{GRANDES PRECIPITACIONES, INUNDACIONES EXTREMAS Y VARIABILIDAD HIDROCLIMÁTICA}

\section{1. Sequías y riesgos: el gran aporte mexicano}

El predominio de sequías extremas en el territorio mexicano ha incidido en la elección de las temáticas a desarrollar por los investigadores, quienes se han interesado (salvo en los últimos años) en mayor medida por los estudios relacionados con este fenómeno. Asimismo, hemos observado una mayor diversidad de ejes 
teórico-metodológicos en la literatura mexicana que hemos revisado, que en la del resto de países.

Recorriendo los trabajos desarrollados en ese país encontramos interesantes análisis sobre los antecedentes de la climatología histórica mexicana. Es el caso del trabajo de García-Acosta (1992) o el de Garza Merodio (2006). Este último autor divide en dos grandes grupos a los escritores mexicanos que se han dedicado al estudio de temas climáticos, desde la Historia o en el marco de otras disciplinas afines como la Geografía. Por un lado, enumera a aquellos especialistas que habrían trabajado dentro de lo que podríamos denominar «estrictamente» climatología histórica3; mientras que, por otro lado, reconoce la existencia de un conjunto de científicos sociales (entre los que menciona a Florescano, 1969; García-Acosta, 2006; O'Hara \& Metcalfe, 1995) que propusieron diferentes interpretaciones de procesos históricos. A estos últimos se incorporan eventos climáticos, como factor explicativo, aunque con diferencias metodológicas y teóricas. Es así que este segundo grupo de autores focaliza los análisis sobre las anomalías climáticas (definidas, en general, desde la interpretación histórica y no desde los métodos cuantitativos) y además prioriza el rol social de los llamados «desastres naturales». Tanto en México como en la mayoría de los países que conforman nuestra área de estudio, se observa una gran cantidad de trabajos que eligen el estudio de casos como método de investigación. Por lo general, se trata del análisis de una sequía o de una inundación extraordinaria interpretada desde la noción de riesgo, desastre y vulnerabilidad. En ese contexto es que se realizan una serie de análisis que leen las problemáticas socioambientales desde el punto de vista de las Ciencias Sociales. Es el caso de los trabajos de Aboites Aguilar \& Camacho Pichardo (1996) y García Hernández (1997) sobre las sequías en México, los cuales forman parte de dos publicaciones coordinadas por García-Acosta. Estas últimas constituyeron un hito en este tipo de estudios en América Latina.

En los estudios pioneros como el de Castorena et al. (1980) o el de Metcalfe (1987) se pueden rastrear metodologías o ejes temáticos consolidados a lo largo de los últimos años, como la generación de series de precipitaciones y temperatura. En ese grupo de estudios tradicionales sobre las sequías mexicanas, hay que mencionar los trabajos que surgen con fuerza durante la década de 1990. Es el caso de la publicación de Liverman (1990) o el ya clásico de Florescano \& Swan (1995), en los cuales los autores sistematizan algunas de las principales fuentes e interpretaciones sobre estas sequías. Estos trabajos son contemporáneos y coinciden desde el punto de vista temático con las publicaciones de O'Hara \& Metcalfe (1995), Tortolero (1996), Endfield \& O'Hara (1997) y Metcalfe et al. (2002).

En la Universidad Autónoma de San Luis Potosí también se han producido progresos en este campo. Además de los estudios de Martínez Chaves et al. (2010) y Ávalos Lozano et al. (2014), hay que mencionar el trabajo

3 En este grupo, el autor destaca la labor de García \& Hernández (1988; 1992), García \& Vidal (1981), Vidal \& García (1980) y Jáuregui (1979). Entre los autores que no son mexicanos, destaca el trabajo de Endfield (2007). 
de Contreras Servín (2005), quien estudia las sequías acaecidas en el siglo XIX en diferentes áreas de México. Empleando una perspectiva propia de la geografía histórica, concluye que estos eventos tuvieron indudables repercusiones económicas, políticas y sociales, las cuales afectaron decisivamente la marcha del país en ese siglo.

Por su parte, García-Acosta et al. (2003) han elaborado un catálogo que contiene una cantidad sin precedentes de datos de sequías históricas en el centro de México (1450-1900). En un trabajo posterior, se utilizó dicha información para compararla con una serie derivada de anillos de árboles y se encontró una importante relación entre los años de fuertes sequías y los años de ocurrencia de El Niño (Mendoza et al., 2005).

Además, hay que mencionar un trabajo clave que estudia, a partir de fuentes históricas, las sequías registradas en la península de Yucatán, ocurridas a lo largo de cuatro siglos (de 1502 a 1900) y las relaciona con sequías modernas (registradas instrumentalmente), utilizando forzantes como la Oscilación Multidecadal del Atlántico (AMO-Atlantic Multi-decadal Oscillation) y el Índice de Oscilación Sur (SOI-Southern Oscillation Index). Se obtuvo una correlación entre las sequías de Yucatán y la fase fría de la $\mathrm{AMO}$, mientras que la influencia de la SOI fue menos clara. También se relacionaron las sequías históricas con la actividad solar y se encontró una vinculación importante (Mendoza et al., 2007).

Por otra parte, si bien las inundaciones no han acaparado tanta atención como las sequías, se puede verificar su presencia en algunos trabajos, como el de Boyer (1975) y el de Musset (1992). Escobar Ohmstede escribió en 1997 acerca de las inundaciones en San Luis Potosí durante 1887.

En los últimos años ha tomado notoriedad otra serie de autores, como es el caso de Richardson Gill (2001), quien estudia el territorio maya, o del propio Garza Merodio (2002; 2006), quien ha elaborado índices climáticos correspondientes a las sequías ocurridas en la cuenca de México desde fines del siglo XVI hasta mediados del siglo XVIII. Esto, a través del análisis de las ceremonias pro pluvia (rogativas) y buscando al mismo tiempo señales de la PEG (Pequeña Edad Glacial) en la región.

Coincidimos con este autor cuando destaca que el estudio del clima durante períodos largos y a una escala regional, utilizando fuentes documentales para desarrollar series relativamente continuas y homogéneas, «ha sido poco utilizad[o] en México hasta fines del siglo XX» (Garza Merodio, 2002: 106). Advierte, además, que los antecedentes más numerosos tuvieron como finalidad considerar la vinculación entre procesos socioeconómicos y eventos climáticos, y más bien localizados espacial y temporalmente.

Sin embargo, en los últimos años han aparecido importantes contribuciones que se alejan de lo señalado por este autor. Destaca el trabajo de Fernández Tejedo (2012), quien, ya habiendo publicado con autoras clásicas sobre el tema (e. g. Endfield et al., 2004), realiza una exhaustiva puesta en valor de los antecedentes de la región al analizar la relación entre el cambio climático y las inundaciones en el Bajío mexicano en el siglo XVIII. Estas fuentes, además de 
brindar información acerca de las sequías e inundaciones catastróficas, aportan datos empíricos acerca del uso y abuso de técnicas agrícolas hidraúlicas. Dan cuenta, a su vez, de las consecuencias socioeconómicas que provocaron el empleo de dichas técnicas, que sumadas a las condiciones climáticas, tuvieron una repercusión negativa sobre los ecosistemas de la región.

\section{2. Los casos sudamericanos}

Más allá del caso mexicano, desde principios de la década de 1980 se han desarrollado trabajos sobre climatología histórica en otras regiones de Latinoamérica desde perspectivas muy variadas. Estos comprenden trabajos básicos que abarcan desde la simple enumeración de eventos y desastres naturales hasta la ejecución de complejos estudios de climatología histórica. Sobre esta última vertiente se pueden mencionar, para el caso de Chile, los trabajos de Ortlieb $(1994 ; 1995 ; 2000)$ que se tratarán en el siguiente apartado. Por su parte, Urrutia de Hazbún \& Lanza Lazcano (1993) recopilaron la ocurrencia de catástrofes en Chile, pero la carencia de referencias exactas sobre las fuentes le resta valor a su trabajo.

Para el caso de Colombia debemos mencionar a Pabón Caicedo (2006) y a Mora Pacheco (2015); para el Perú, a Carcelén Reluz (2009); y para Bolivia, a Gioda \& Prieto (1999a). Si nos referimos a Ecuador, Terneus \& Gioda (2006) y Domínguez Castro et al. (2018) son referencias muy importantes. Estos últimos estudiaron las grandes sequías y los extremos húmedos en Quito en los últimos 400 años. Dicho estudio se realizó a partir de una larga serie instrumental (1891-2015) y de las rogativas pro serenitate, en la época lluviosa, y pro pluvia, más frecuentes en enero y febrero cuando el agua solía escasear. Los autores resuelven que las sequías se han intensificado, en frecuencia y duración, desde mediados del siglo XX. También mencionan los períodos más graves de sequías, siendo el más severo de todos el ocurrido entre 1692 y 1701.

Entre las numerosas reuniones científicas organizadas en los Andes centrales, podríamos mencionar el Simposio Internacional «15.000 años de clima y hombre en los Andes Centrales: enfoques paleoclimatológicos y arqueológicos», desarrollado en Lima en agosto de 2013 (Ortlieb, 2013).

Para el caso de Brasil, destacan Araki e Hidalgo Nunes, quienes han realizado la reconstrucción y la interpretación histórica del clima en el estado de San Pablo (Araki \& Hidalgo Nunes, 2009; Araki, 2012). Este trabajo sistematizó los datos de diversas fuentes documentales desde el período colonial, tanto de temperaturas como de precipitaciones, para comprobar la hipótesis de un aumento de las temperaturas que se produjo en dicho estado en el transcurso de los últimos siglos. En la región Nordeste de Brasil, los trabajos se concentran en las sequías. Por ejemplo, Palacios (1996) contradice la idea generalizada de que han sido las recurrentes sequías en el Nordeste brasileño las que han provocado el deterioro social de la región, minimizando las responsabilidades políticas. En dicha línea se inscribe el trabajo de Da Guia Santos Gareis et al. (1997), quienes interpretan la 
vulnerabilidad de la población del Sertao y demuestran el rol preponderante de los Estados coloniales en la conformación de estos desastres naturales. En un sentido similar, Villa (2000) da cuenta de los problemas y de los desaciertos políticos en la conformación de estas problemáticas socioambientales.

Davis (2006) analiza el rol de las graves sequías y de los eventos ENOS (El Niño Oscilación Sur) en el contexto de las consecuencias negativas de las políticas económicas coloniales de fines del siglo XIX, las cuales produjeron, entre otros problemas, el aumento de hambrunas a escala mundial. En el caso de Brasil, este autor explica cómo la gran depresión económica de 1873 afectó a los campesinos del Nordeste, ya muy golpeados por las recurrentes sequías 4 . Hemos rastreado también un interesante trabajo de Ferreira da Silva et al. (2012), en el cual los autores estudiaron la ocurrencia de granizo en el estado de Paraná utilizando como fuente los periódicos entre 1960 y 2009. Llama la atención, en el sur de Brasil, la carencia de trabajos sobre las inundaciones históricas de los caudalosos ríos que atraviesan esos territorios.

En Argentina, los investigadores se han dedicado en gran parte a los estudios de variabilidad climática relacionados con las precipitaciones regionales y los caudales de los principales ríos de la región. Esto implica la construcción de series de eventos hidroclimáticos que permitan determinar cambios y continuidades en el clima sudamericano y su incidencia sobre la sociedad y la economía. Desde esta última perspectiva destacan trabajos como el estudio de la relación entre la caída de granizo, la ocurrencia de heladas en Mendoza y su importancia en la vitivinicultura regional desde fines del siglo XIX, para el cual se utilizó información periodística (Prieto, Soria et al., 1995). El trabajo de Gil-Guirado et al. (2016) representa particular interés pues analiza la adaptación a las sequías extremas y a las inundaciones, desde el siglo XVII, a partir del Índice perceptual de cambios en el riesgo climático (PICCR-Perceptual Index for Changes in Climate Risk), comparando - entre otros datos - la serie hidrológica del río Mendoza y el río Segura (Murcia, España).

En esta misma línea, se puede citar el trabajo pionero de Prieto \& Richard Jorba (1991) acerca de las inundaciones en la Cuenca del Plata y en la región Noroeste Argentino (NOA), y de sus consecuencias socioeconómicas durante los siglos XVI, XVII y XVIII. Prieto, basado en ese trabajo, analizó las diferentes respuestas culturales frente a las inundaciones, desplegadas por indígenas y españoles durante la Colonia (Prieto, 2011).

En cuanto a la elaboración de series hidroclimáticas, se han determinado las fluctuaciones en la escorrentía de algunos de los ríos argentinos, en especial del río Mendoza (Prieto, Herrera \& Dussel, 1999; Prieto, Herrera, Castrillejo et al., 2001; Prieto \& Rojas, 2012; véase fig. 1).

4 La respuesta estatal para estas crisis sociales de subsistencia apuntó a una intensificación de medidas que fomentaban el mercado global, mientras desestructuraban las economías locales y los sistemas de reciprocidad que las culturas regionales habían generado para paliar crisis ambientales y sociales. Un ejemplo extremo de ello es la brutal represión en Canudos (Davis, 2006). 


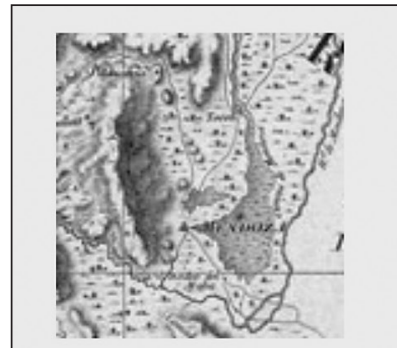

1794 Espinosa y Bauzá

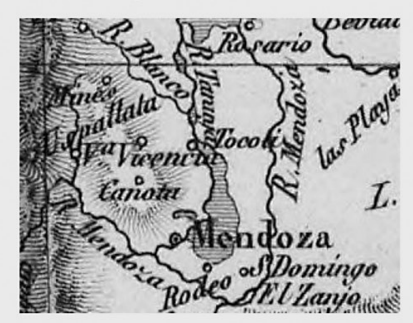

1826 Brue

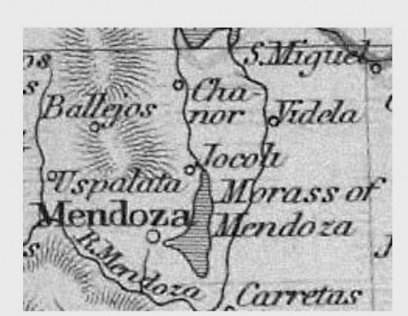

1851 Martin and Tilles

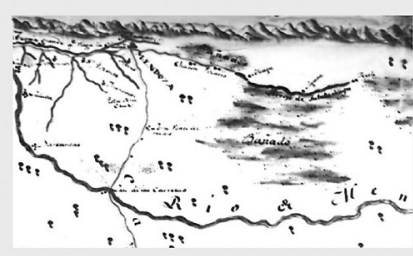

1800 Serra Canals

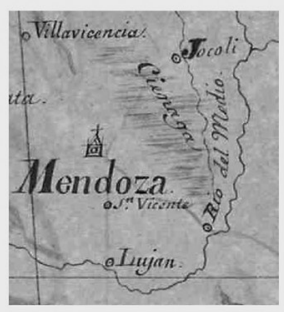

1837 Álvarez Condarco

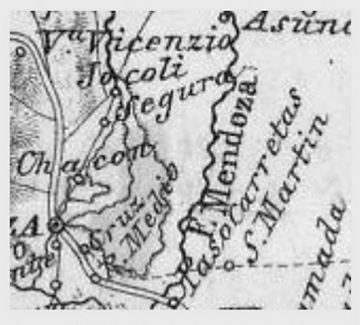

1854 Arrowsmith et al.

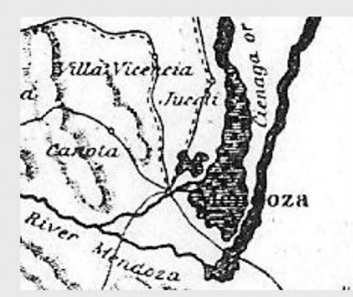

1824 Hibbert

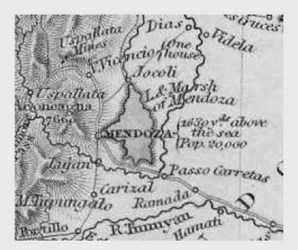

1840 Society for the Diffusion of Useful Knowledge

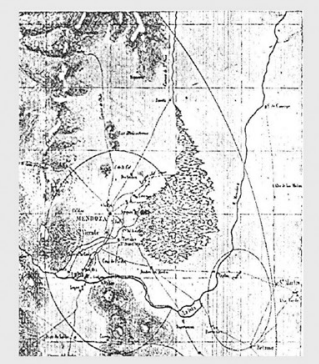

1861 Díaz

Figura 1 - Mapas de la Ciénaga del Bermejo (Mendoza-Argentina) en diferentes años, entre 1794 y 1861, donde figura la variación en su volumen de acuerdo con las fluctuaciones del río Mendoza (Prieto \& Rojas, 2012: 957, fig. 5)

Las series del río Mendoza, derivadas del análisis documental, han sido vinculadas también a diferentes procesos ambientales, como los sucesivos momentos de avance y retroceso de los glaciares en la Cordillera de los Andes. Ello ha sido posible gracias a la disponibilidad de series paleoambientales originadas a partir del análisis de los anillos de árboles (Villalba, 1994) y de datos geológicos (Espizúa, 2003).

Se han realizado, además, estudios sobre variabilidad climática mediante el desarrollo de series de sequías y de importantes eventos de precipitaciones desde Jujuy, Tucumán, Santiago del Estero, Santa Fe y Corrientes, en el norte de Argentina, hasta Mendoza y Córdoba, en el centro-oeste y centro de ese país, respectivamente (Prieto, Herrera \& Dussel, 2000). Esto significa una exhaustiva exploración y extracción de la información de los archivos documentales, mediante análisis de contenido (Prieto, Gallego et al., 2005). Posteriormente, se ha procedido 
a la construcción de índices ordinales de precipitación que pueden incluir, por lo menos, cinco clases: de muy húmedo $(+2)$ a muy seco $(-2)$. Con similar metodología se han realizado cronologías de precipitaciones en Santiago del Estero y de caudales del río Salí-Dulce (que atraviesa esa ciudad argentina), principalmente con la información provista por las Actas Capitulares desde el siglo XVII, las cuales consignan, además, los sucesivos cambios de curso de ese río (Herrera et al., 2011). Para la misma región, se ha construido una serie hidroclimática del río Bermejo (también desde el siglo XVII) en la cual se puede observar el incremento de la frecuencia de inundaciones desde 1800 y la disminución de sequías severas desde 1891 (Prieto \& Rojas, 2015). Para calibrar y verificar las series obtenidas se aplicaron operaciones estadísticas desarrolladas por Neukom et al. (2009).

No podemos dejar de mencionar el primer trabajo conocido en Argentina realizado mediante el uso de documentación de archivo. Se trata de una serie de datos elaborada a partir de la información sobre sequías que aparecía semanalmente en las Actas del Cabildo de Buenos Aires (Politis, 1984). La tarea de construir índices ordinales sobre el clima de la provincia de Buenos Aires ha sido escasamente continuada por otros especialistas. Un ejemplo de lo anterior es el trabajo de Deschamps et al. (2003) quienes, continuando lo realizado por Politis, analizaron fuentes históricas y utilizaron como proxy información arquitectónica de Buenos Aires en el período tardocolonial. Durante ese período, las casas respondían a modelos de techo plano compatible con espacios de escasas lluvias. A partir del siglo XIX se produjo un cambio tanto en la arquitectura como en la vegetación, como consecuencia de un continuo aumento de las precipitaciones e inundaciones (Deschamps et al., 2003). Debemos mencionar, además, la publicación del trabajo de Banzato (2016), el cual relaciona las recurrentes inundaciones de la provincia de Buenos Aires con políticas públicas, discursos y aspectos económicos. Para el Noroeste Argentino, López de Albornoz (1997) vinculó las sequías y las heladas en el Tucumán colonial con las crisis agrícolas y las epidemias regionales. En la misma región, Tasso (2011) historiza la gran sequía de 1935-1937 en Santiago del Estero, la cual desató la hambruna más notable en la historia contemporánea de esta provincia argentina.

\section{3. El Caribe y los ciclones}

En los países del Caribe, los historiadores del clima se han abocado principalmente al estudio de los huracanes, dada la importancia de este fenómeno en la región. Aunque se analiza con frecuencia el comportamiento de los huracanes más violentos de forma particular, ha surgido la necesidad de estudiar la actividad de los ciclones tropicales a largo plazo, para verificar si han aumentado su frecuencia e intensidad como consecuencia del cambio climático. La documentación histórica ha permitido describir y analizar la frecuencia de los ciclones con datos acerca de su ocurrencia desde principios del siglo XVI (Walsh \& Reading, 1991; Rappaport \& Fernández-Partagás, 1997). También existen recopilaciones. GarcíaHerrera et al. (2004) realizaron un importante trabajo de análisis de huracanes 
históricos del Caribe usando cuadernos de bitácora de barcos españoles e ingleses. Fernández-Partagás \& Díaz (1996) también se han dedicado a estudiar la frecuencia de los huracanes durante la segunda mitad del siglo XIX.

Fuera del tema de los ciclones tropicales del Caribe, también hemos encontrado otras temáticas desarrolladas por los investigadores de esa región, como el estudio sobre el clima y la variabilidad climática en Costa Rica realizado por Amador (2002), quien utilizó antiguos datos instrumentales del siglo XIX. Asimismo, existen algunos trabajos, como el de Cocco Quezada (1992), sobre las sequías en República Dominicana. Muchos de los trabajos centroamericanos están basados en compilaciones de fuentes y datos, aunque usualmente algunos no se han publicado y otros no han sido analizados de forma sistemática.

\section{EL NIÑO OSCILACIÓN SUR}

Es bien sabido que la Costa norte del Perú y el sur de Ecuador son las áreas afectadas de manera más directa por el aumento de la temperatura del mar durante un evento El Niño. Este forzante climático (El Niño Oscilación Sur) ha captado la atención de numerosos investigadores que buscan determinar su relación con las sequías e inundaciones históricas.

En el presente apartado se ha puesto un particular énfasis en aquellas regiones donde ENOS es reconocido como un factor significativo que influye en la variabilidad interanual de las precipitaciones, desde su abundancia hasta su ausencia total, como las sequías. A escala latinoamericana, el trabajo de Caviedes (2001) explica la conexión entre los eventos ENOS y las tormentas anómalas en el Perú, Ecuador y Chile, así como también las sequías en el altiplano sudamericano, el mexicano y el Nordeste de Brasil.

México —además de Colombia, Venezuela y Guyana, donde El Niño potencia los años secos (Poveda \& Mesa, 1996) - , las tierras altas de los Andes centrales (altiplano) y el Nordeste de Brasil están incluidos en el grupo de los países y de las regiones con sequías, en ocasiones extremas, cuando se produce un evento El Niño. Por el contrario, las áreas costeras del Ecuador y del Perú, el sureste del subcontinente sudamericano (noreste de Argentina, Paraguay y sureste de Brasil) y la región de Chile Central sufren fuertes precipitaciones pluviales (o nivales en la Cordillera de los Andes).

Numerosos registros proxy de ENOS han sido desarrollados durante las décadas pasadas. Estos incluyen anillos de árboles, testigos de hielo y documentos de archivo, entre otros, los cuales notifican lluvias inusuales a lo largo de la Costa norte del Perú (Díaz \& Markgraf, eds., 1992; Markgraf, 2000). Se han realizado cronologías de EI Niño desde fines del siglo XIX en la región, dado que esta es particularmente sensible a la fase cálida del fenómeno. En años recientes, la más completa cronología documental referida a ENOS fue aquella publicada por Quinn et al. (Quinn et al., 1978; Quinn \& Neal, 1983; Quinn et al., 1987; Quinn, 1992) desde comienzos del siglo XVI, utilizando en su mayoría fuentes secundarias, algunas veces controversiales. 
Hamilton \& García (1986), continuando el temprano trabajo de Quinn, identificaron posibilidades adicionales para fuertes eventos El Niño. Ortlieb (2000) revisó la cronología realizada por Quinn, reinterpretó las fuentes sudamericanas usadas por dicho autor, verificó la confiabilidad de los reportes, la intensidad de los eventos, la calidad de los datos y su significado, y detectó algunas incertidumbres. Huertas Vallejos $(1993 ; 2001)$ analizó el impacto social y económico de El Niño en el Perú durante un amplio período histórico. García-Herrera et al. (2008) desarrollaron una nueva cronología de El Niño utilizando registros documentales del norte del Perú entre los años 1550 y 1900. Esta cronología está construida con fuentes primarias de la ciudad de Trujillo (Perú), del Archivo General de Indias en Sevilla (España) y del Archivo General de la Nación de Lima (Perú), e incluyen, en algunos casos, material suplementario proveniente de la literatura previamente publicada.

Además, tomando como base los registros del Galeón de Manila —que navegaba con regularidad entre Acapulco, en México, y Filipinas - se ha estudiado el comportamiento de ENOS en el Océano Pacífico meridional durante el siglo XVI (García et al., 2001).

En Chile, a pesar de que la señal de El Niño es clara y se traduce en copiosas Iluvias — sobre todo en la región de Chile Central — existen menos estudios de este tipo (Prieto, Solari et al., 2012). Aunque algunos historiadores ya desde el siglo XIX (Vicuña Mackenna, 1970) se han dedicado a recopilar acontecimientos catastróficos relacionados con inundaciones y grandes crecidas, fue Taulis (1934) quien sistematizó la información correspondiente a Santiago, la capital chilena, y completó los registros faltantes hasta 1934, utilizando fundamentalmente las Actas Capitulares de esa ciudad. Se debe atribuir a Ortlieb (1994) la revisión de los registros consignados por estos autores, relacionando las lluvias de la región de Chile Central con la ocurrencia de años El Niño Oscilación Sur. Este mismo autor realiza una compilación muy detallada de las lluvias en el norte chileno durante el siglo XIX (Ortlieb, 1995). El trabajo de Aceituno et al. (2009) constituye un estudio reciente y más específico que relaciona el fenómeno de El Niño de los años 1877 y 1878 con sus efectos económicos, sociales y políticos en todos los países sudamericanos alcanzados por las repercusiones de este fenómeno (incluido Chile).

Los estudios sobre el clima de los Andes argentino-chilenos y su conexión con ENOS se han desarrollado mayormente en la zona sur de Sudamérica, sobre todo en Mendoza, Argentina, gracias al estudio de anillos de árboles, documentos de archivo y periódicos, relacionando los años de mayores precipitaciones nivales con la ocurrencia de ENOS en el Océano Pacífico y las precipitaciones en Chile (Masiokas et al., 2012). También se relacionó con el Niño la serie de caudales del río Mendoza cuyas fuentes se encuentran precisamente en la vertiente oriental de los Andes centrales argentino-chilenos (Prieto, Herrera \& Dussel, 1999; Prieto, Herrera, Castrillejo et al., 2001).

Entre los ríos del noreste de Argentina, íntimamente ligados a la ocurrencia de El Niño, destaca el río Paraná. Se debe mencionar la reconstrucción de su caudal 
entre 1590 y 1805 (crecidas e inundaciones), realizada utilizando documentación histórica de las ciudades de Santa Fe y Corrientes, principalmente las Actas Capitulares de esas ciudades y los informes de Jesuitas y Franciscanos (Prieto, 2007; 2009). Tomando como base el trabajo de Ortlieb (2000), se relacionaron las mayores crecidas del Paraná inferior con los eventos ENOS.

En cuanto a las sequías relacionadas con este fenómeno en Bolivia, existen series de precipitaciones de algunas unidades administrativas pertenecientes a la puna boliviana, en las cuales se hace hincapié en la ocurrencia de sequías relacionadas con El Niño. Destacan los trabajos sobre Potosí y La Paz (Gioda \& Prieto, 1999a; 1999b).

\section{EL CLIMA COSTERO Y LOS GLACIARES}

Debemos señalar que, a diferencia de Europa, en Sudamérica son escasos los dibujos y pinturas antiguas sobre glaciares. Esta es una de las razones por las que son excepcionales los trabajos sobre glaciares realizados con este tipo de documentación histórica (para el Perú y Ecuador véase Ames Marquez \& Francou, 1995; para Ecuador, Francou, 2004). Un ejemplo para Ecuador, pero desde una perspectiva etnoecológica, es el trabajo de Rhoades (2008). El autor presenta un estudio documentado de la desaparición del glaciar del nevado Cotacachi en Ecuador, utilizando una metodología que combina una serie de fuentes (análisis etnográficos, representaciones visuales, fotografía repetitiva, mapeo crítico por parte de las comunidades locales, investigación de archivos). Este estudio alerta acerca de la falta de investigaciones sociales relacionadas con el cambio climático global, con un enfoque cultural local. En el caso de aquellas investigaciones realizadas sobre la Patagonia continental se han utilizado, por ejemplo, fotografías antiguas para conocer las fluctuaciones del glaciar Perito Moreno (Guerrido et al., 2014).

Con relación al clima costero, la mayoría de los estudios se basan en los avistamientos y las descripciones que realizaban los marinos desde los barcos mercantes durante los siglos XVI y XVII, al cruzar el estrecho de Magallanes o el Cabo de Hornos, para luego remontar la costa chilena y encontrarse con los imponentes glaciares del estrecho y del sur de Chile que se podían observar desde el mar. Es por ello que los cuadernos de navegación de los barcos españoles y de los de otros países europeos contienen valiosa información sobre el clima marítimo austral de Argentina y Chile, sobre todo del estrecho de Magallanes en la estación de primavera-verano entre los años 1520 y 1619, así como descripciones de los cuerpos glaciares que descendían al mar tanto en el estrecho como en las costas australes de Chile (Prieto \& Herrera, 1998; Araneda et al., 2007). Los avistamientos de grandes conjuntos de icebergs desprendidos de la Antártida dirigiéndose a latitudes más bajas se han verificado en la segunda mitad del siglo XVIII, sobre todo en el Océano Austral argentino. Prieto et al. (2004) comprobaron cinco avistamientos de icebergs durante ese período, en formaciones «tipo enjambre», anteriores a los consignados por el capitán Cook (1772-1775). 
En el sur de Mendoza, Cobos \& Boninsegna (1983), basados en documentos y fotos antiguas, compararon la variabilidad del caudal del río Atuel a partir del análisis de anillos de árboles de la zona chilena con los avances y retrocesos de glaciares en el sur de Mendoza. Prieto (1986) estudió un evento de endicamiento glaciar (surge) sobre el río Plomo (Mendoza, Argentina) en 1786, similar a los sucedidos en los años 1934 y 1984 utilizando documentación del AGI.

\section{CLIMA, ETNOHISTORIA Y ETNOGRAFÍA}

Existen numerosos ejemplos de investigaciones que, desde diversas perspectivas y en ocasiones combinando metodologías que implican un abordaje multidisciplinar, se preocuparon por reconstruir parte de la historia climática y ambiental de una región en un tiempo determinado. Para ello, no solamente recurrieron a la construcción y/o uso de series climáticas actuales y pasadas, sino que, además, integraron el estudio de las percepciones, las interpretaciones y/o las respuestas que determinados pueblos ensayaron frente a las fluctuaciones ambientales.

El estudio de Florescano Mayet et al. (1980), por ejemplo, propuso reconstruir la historia, las características y los efectos de las sequías en el Bajío mexicano. Para los tiempos prehispánicos utilizaron fuentes indígenas y crónicas antiguas que señalan que en los años en que «llovía fuego» o no había suficiente precipitación se perdían cosechas de maíz, bajaba el nivel de la laguna de México y las chinampas quedaban encalladas en la tierra (Florescano Mayet et al., 1980: 747-748). Este trabajo se convierte en una de las primeras investigaciones que, mediante el uso de documentación etnohistórica y registros históricos, describe este fenómeno. Hay que resaltar nuevamente el aporte de Richardson Gill (2001), quien, a través de un estudio interdisciplinario, incorpora a la etnohistoria para desarrollar su hipótesis en torno a la importancia de las sequías en la decadencia de la cultura maya. Carcelén Reluz (2007) describe como muy constante la existencia de manifestaciones religiosas producidas como efecto de fenómenos climáticos. Su objetivo, sin embargo, es demostrar que estos abogan por una búsqueda generalizada de estabilidad, invocando unos al Dios y santos católicos, y otros a los apus y huacas andinas. Otros historiadores y geógrafos, también a partir de una metodología etnohistórica, han indagado acerca de la relación entre el surgimiento de epidemias, sequías e inundaciones ocurridas entre las poblaciones indígenas del Alto Perú y su impacto económico (Prieto \& Herrera, 2002; Prieto \& Rojas, 2012). Desde un abordaje netamente etnohistórico, se han estudiado los efectos demográficos y económicos que las sequías tuvieron sobre la población indígena y española en el siglo XVIII, considerándolos como un factor explicativo más dentro de un conjunto de variables (Tandeter \& Wachtel, 1984). Calvo (2016), en México y Cartagena de Indias (entre los años 1690 y 1692), combina la información propia de la demografía histórica con los datos de la producción de semillas y precios (hambrunas, bonanzas, epidemias, etc.) y las fluctuaciones climáticas a nivel planetario. Gil Montero junto con dendrocronólogos analizaron algunas sequías ocurridas en el siglo XIX desde el 
punto de vista de la demografía indígena y revisaron los libros de defunciones de los pueblos más afectados (Gil Montero et al., 2010). Pentimalli de Navarro \& Rodríguez Ostria (1988) retomaron el tema para analizar los efectos sociales, económicos y demográficos de la terrible sequía de los años 1877-1878 en Sucre y en otras ciudades de Bolivia. Algunos estudios se enfocaron en describir cómo ciertas sociedades respondieron a la necesidad de reducir su vulnerabilidad ante las sequías. Este es el caso de Padilla Lozoya (2016), quien analiza el surgimiento de una sociedad vulnerable y la manera como responde esta ante las amenazas climáticas, en San José del Cabo San Lucas, Baja California Sur.

Por su parte, Gascón ha realizado aportes desde el enfoque de la etnoclimatología, en colaboración con Caviedes (Gascón \& Caviedes, 2012; 2014). Ellos la definen como «el estudio de las formas en que los indígenas reaccionaban y reaccionan actualmente ante las fluctuaciones climáticas, utilizando el conocimiento adquirido sobre el clima local a través de sus observaciones y conocimientos empíricos del medio natural» (Gascón \& Caviedes, 2014: 141-142). Los autores desarrollan, en dichos trabajos, una correlación entre cambios climáticos, disponibilidad de recursos en el ambiente y relaciones interétnicas (entre españoles e indígenas) en la frontera sur de las pampas argentinas, hacia el siglo XVIII.

En esta misma línea hay autores que relacionan cambio climático y pueblos indígenas, pero orientados hacia el conocimiento etnoclimático tradicional, obtenido a partir de las percepciones, interpretaciones y respuestas de estos pueblos frente al cambio climático. Entre estos trabajos podemos mencionar el de Fernández-Llamazares et al. (2015) en la Amazonía boliviana actual y el de Sánchez-Cortés \& Lazos Chavero (2011) en la comunidad Zoque de Chiapas, México. Los autores incorporan, a su vez, registros de precipitaciones, temperaturas y datos sobre erupciones volcánicas. En este sentido, Echeverri (2009) contrasta los registros de temperatura, precipitaciones, niveles fluviales y estacionalidad con el conocimiento indígena del calendario ecológico en la Amazonía colombiana, entre los años 1970 y 2007.

\section{CONCLUSIONES}

El desarrollo de la climatología histórica latinoamericana denota un importante crecimiento en las últimas décadas, aunque bastante centrado en ciertas regiones, temáticas y áreas disciplinares.

Repasando los principales antecedentes, destaca una mayor producción desarrollada en México, Argentina y la costa pacífica de Sudamérica (Colombia, Ecuador, Perú y Chile). En ese contexto, se pueden identificar diferentes ejes temáticos predominantes, como son, por una parte, los estudios de series climáticas (casi exclusivamente de precipitaciones y caudales), generalmente relacionadas con otros proxy records (instrumentales y dendrocronológicos principalmente) y con potentes estudios en México, el Cono Sur y los Andes centrales. En síntesis, desde las extensas series de eventos ENOS recopilados y calibrados por 
diversos autores —en Perú, Chile y Ecuador - hasta la reconstrucción de las precipitaciones y del caudal de los ríos de Argentina — desde el siglo XVII hasta el siglo XX - creemos que se ha avanzado en gran medida en el desarrollo de este campo de estudio, desde perspectivas más cuantitativas — en sintonía con las desarrolladas en Europa por investigadores de la talla de Pfister o Brázdil-. Estas perspectivas incorporan metodologías que tienden a asegurar la confiabilidad de los datos extraídos de archivos, incluyendo la calibración de las series construidas en función de la información instrumental. A su vez, permiten la contrastación de otras series climáticas provenientes de huellas que han dejado las variaciones climáticas en otras fuentes, como los sedimentos lacustres o los anillos de árboles.

Asimismo, la labor desarrollada para interpretar las relaciones entre diferentes procesos de variabilidad climática y la adaptación y/o vulnerabilidad social (principalmente en México, Brasil y los Andes centrales) ha sido intensa. En sintonía con este marco referencial hay que destacar los estudios que rescatan la agencia indígena y muestran las diversas concepciones sobre «lo natural», con fuertes implicancias en las prácticas «ambientales» y, por ende, en los abordajes académicos que buscan captar dichas complejidades.

Las sequías del Nordeste brasilero son otra temática recurrente que ha permitido comprender la fuerte vinculación (usual en muchas regiones de Latinoamérica) entre la dispar apropiación de los recursos naturales y los problemas ambientales. Por último, en muchos países han existido recopilaciones de eventos históricos extremos, aunque con insuficiente tratamiento teórico/metodológico. Se pueden observar entonces extensas listas de eventos, pero sin un marco de discusión conceptual, o sin una metodología ordenada que permita la comparación con otras series y estudios.

Además de su difusión como disciplina y la profundización teórico-metodológica sobre líneas desarrolladas hasta el momento, vemos en el horizonte otros desafíos. Nos referimos a la discusión e interacción con otras disciplinas, campos de estudio y problemáticas de los que intentamos brindar un panorama en el último apartado. En esa dirección también es necesaria una mayor integración y un mayor debate (teórico y metodológico) entre las diferentes vertientes de estudio propias de la climatología histórica y otras disciplinas académicas como la historia ambiental, la ecología política, la sociología ambiental, la Antropología y la Geografía.

\section{Agradecimientos}

Agradecemos al Consejo Nacional de Investigaciones Científicas y Técnicas (CONICET) por financiar esta investigación, a Katherinne Mora Pacheco por sus valiosos comentarios, a Marcelo Giraud, Erik Marsh y María Eugenia Solari por las traducciones del resumen al francés y al inglés, respectivamente, y a los revisores por sus valiosos aportes. 


\section{Referencias citadas}

Archivo General de Indias (AGI), Audiencia de Buenos Aires, legajos: 21, 73, 99, 107, 109, 383, 586, 587, 590; Indiferente General, legajos: 1559, 1528.

ABOITES AGUILAR, L. \& CAMACHO PICHARDO, G., 1996 - Aproximación al estudio de una sequía en México. El Caso de Chapala-Guadalajara (1949-1958). In: Historia y Desastres en América Latina, volumen I (V. García-Acosta, coord.): 259-291; Santafe de Bogotá: Red de Estudios Sociales en Prevención de Desastres en América Latina (LA RED), Centro de Investigaciones y Estudios Superiores en Antropología Social (CIESAS).

ACEITUNO, P., PRIETO, M. R., SOLARI, M. E., MARTíNEZ, A., POVEDA, G. \& FALVEY, M., 2009 - The 1877-1878 El Niño episode: Associated impacts in South America. Climatic Change, 92 (3-4): 389-416.

AMADOR, J., 2002 - Clima y variabilidad climática en Costa Rica a través de información histórica del siglo XIX. In: Estudios sobre historia y ambiente en América, II: Norteamérica, Sudamérica y el Pacífico (B. García Martínez \& M. R. Prieto, comps.): 37-54; México, D. F.: El Colegio de México, Instituto Panamericano de Geografía e Historia (IPGH).

AMES MARQUEZ, A. \& FRANCOU, B., 1995 - Cordillera Blanca. Glaciares en la Historia. Bulletin de l'Institut Français d'Études Andines, 24 (1): 37-64.

ARAKI, R., 2012 - A História do Clima de São Paulo. Campinas, São Paulo: Universidade Estadual de Campinas (UNICAMP), Instituto de Geociências. Tesis de doctorado.

ARAKI, R. \& HIDALGO NUNES, L., 2009 - Ancient Natural Disasters triggered by severe weather in São Paulo, Brazil. In: $5^{\text {th }}$ European Conference on Severe Storms, 12 16 October 2009, Landshut, Germany.

ARANEDA, A., TORREJÓN, F., AGUAYO, M., TORRES, L., CRUCES, F., CISTERNAS, M. \& URRUTIA, R., 2007 - Historical records of San Rafael glacier advances (North Patagonian Icefield): another clue to "Little Ice Age" timing in Southern Chile. The Holocene, 17 (7): 987-998.

ARRIOJA DÍAZ VIRRUEL, L. A., 2016 - Clima, plagas y desolación en la provincia de Chiapa, 1768-1772. In: Clima, desastres y convulsiones sociales en España e Hispanoamérica, siglos XVII-XX (L. A. Arrioja Díaz Virruel \& A. Alberola Romá, eds.): 295-321; Alicante: El Colegio de Michoacán, Universidad de Alicante.

ÁVALOS LOZANO, J. A., MEDELLíN MILÁN, P. \& AGUILAR ROBLEDO, A., 2014 - The meaning of climate change in San Luis Potosi, Mexico. In: Actas del VII Simposio de la Sociedad Latinoamericana y Caribeña de Historia Ambiental y XI Jornadas de Investigación y Debate, 15 al 18 de octubre de 2014: 21; Buenos Aires: Centro de Estudios de la Argentina Rural, Universidad Nacional de Quilmes.

BANZATO, G., 2016 - Presupuestos y gastos del estado en la provincia de Buenos Aires para afrontar las inundaciones de los campos, 1870-1930. Revista Uruguaya de Historia Económica, 6 (9): 31-48.

BOYER, R. E, 1975 - La Gran Inundación. Vida y sociedad en la ciudad de México (16291638), 151 pp.; México: Secretaría de Educación Pública.

BRÁZDIL, R., PFISTER, C., WANNER, H., VON STORCH, H. \& LUTERBACHER, J., 2005 Historical Climatology in Europe-The State of the Art. Climatic Change, 70: 363-430.

CALVO, T., 2016 - Algunas historias de granos en medio de fluctuaciones planetarias: México y Cartagena de Indias en 1690-1692. In: Clima, desastres y convulsiones 
sociales en España e Hispanoamérica, siglos XVII-XX (L. A. Arrioja Díaz Virruell \& A. Alberola Romá, eds.): 269-294; Alicante: Universidad de Alicante, El Colegio de Michoacán.

CARCELÉN RELUZ, C. G., 2007 - Idolatría indígena y devoción criolla como respuestas a la variabilidad climática en Lima y Huarochirí durante el siglo XVIII. Investigaciones Sociales, XI (19): 173-188.

CARCELÉN RELUZ, C. G., 2009 - Historia del clima y el medio ambiente en Lima y el Perú central en el siglo XVIII: Problema de investigación y fuentes históricas. Revista de Historia de América, 140: 51-94.

CASTORENA, G., SÁNCHEZ MORA, E., FLORESCANO, E., PADILLO RÍOS, G. \& RODRÍGUEZ VIQUEIRA, L., 1980 - Análisis histórico de las sequías en México Documentación de la Comisión del Plan Nacional Hidráulico, 158 pp.; México, D. F.: Secretaría de Agricultura y Recursos Hidráulicos (SARH), Comisión del Plan Nacional Hidráulico.

CAVIEDES, C. N., 2001 - El Niño in History: Storming Through the Ages, XIV + 279 pp.; Gainesville: University Press of Florida.

COBOS, D. R. \& BONINSEGNA, J. A., 1983 - Fluctuations of some glaciers in the upper Atuel River basin, Mendoza, Argentina. Quaternary South America and Antarctic Peninsula, 1: 61-82.

COCCO QUEZADA, A., 1992 - Análisis de las sequías en la República Dominicana, 10 pp.; Santo Domingo: ACQWeather. Meteorología, clima y desastres. Disponible en http://www.acqweather.com/bibliografica.html.

CONTRERAS SERVÍN, C., 2005 - Las sequías en México durante el siglo XIX. Investigaciones Geográficas, Boletín del Instituto de Geografía, UNAM, 56: 118-133.

CUSHMAN, G. T., 2011 - Humboldtian Science, Creole Meteorology, and the Discovery of Human-Caused Climate Change in Northern South America. Osiris, 26 (1): 19-44.

DA GUIA SANTOS GAREIS, M., DO NASCIMENTO, J. A., MOREIRA, A. F. \& DA SILVA, M. A., 1997 - Aspectos históricos de las sequías en el Nordeste del Brasil colonial (1530-1822). In: Historia y Desastres en América Latina, volumen II (V. García-Acosta, coord.): 103-132; Lima: Red de Estudios Sociales en Prevención de Desastres en América Latina (LA RED), Centro de Investigaciones y Estudios Superiores en Antropología Social (CIESAS), Intermediate Technology Development Group (ITDG).

DAVIS, M., 2006 - Los holocaustos de la Era Victoriana tardía. El Niño, las hambrunas y la formación del Tercer Mundo, 448 pp.; Valencia: Universitat de València.

DESCHAMPS, J. R., OTERO, O. \& TONNI, E. P., 2003 - Cambio climático en la pampa bonaerense: las precipitaciones desde los siglos XVIII al XX, 18 pp.; Buenos Aires: Universidad de Belgrano. Área de Estudios Agrarios.

DÍAZ, H. F. \& MARKGRAF, V. (eds.), 1992 - El Niño. Historical and Paleoclimatic Aspects of the Southern Oscillation, XII + 476 pp.; Cambridge, Nueva York: Cambridge University Press.

DOMíNGUEZ CASTRO, F., GARCÍA-HERRERA, R. \& VICENTE-SERRANO, S. M., 2018 Wet and dry extremes in Quito (Ecuador) since the 17th century. International Journal of Climatology, 38 (4): 2006-2014.

ECHEVERRI, J. A., 2009 - Pueblos indígenas y cambio climático: el caso de la Amazonía colombiana. Bulletin de l'Institut Français d'Études Andines, 38 (1): 13-28.

ENDFIELD, G. H., 2007 - Climate and Crisis in Eighteenth Century Mexico. The Medieval History Journal, 10 (1-2): 99-125. 
ENDFIELD, G. H., FERNÁNDEZ TEJEDO, I. \& O'HARA, S. L., 2004 - Conflict and Cooperation: Water, Floods, and Social Response in Colonial Guanajuato, Mexico. Environmental History, 9 (2): 221-247.

ENDFIELD, G. H. \& O'HARA, S. L., 1997 - Conflicts Over Water in 'The Little Drought Age' in Central Mexico. Environment and History, 3 (3): 255-272.

ESCOBAR OHMSTEDE, A., 1997 - Las "sequías" y sus impactos en las sociedades del México decimonónico, 1856-1900. In: Historia y Desastres en América Latina, volumen II (V. García-Acosta, coord.): 219-257; Lima: Red de Estudios Sociales en Prevención de Desastres en América Latina (LA RED), Centro de Investigaciones y Estudios Superiores en Antropología Social (CIESAS), Intermediate Technology Development Group (ITDG).

ESPIZÚA, L., 2003 - Holocene glacier fluctuations in the south of Mendoza Andes, Argentina. In: Actas del II Congreso Argentino de Cuaternario y Geomorfología (M. M. Collantes, J. M. Sayago \& L. V. Neder, eds.): 87-92; Tucumán: Ediciones MAGNA publicaciones.

FERNÁNDEZ-LLAMAZARES, Á., MÉNDEZ-LÓPEZ, M. E., DÍAZ-REVIRIEGO, I., McBRIDE, M. F., PYHÄLÄ, A., ROSELL-MELÉ, A. \& REYES-GARCÍA, V., 2015 Links between media communication and local perceptions of climate change in an indigenous society. Climatic Change, 131 (2): 307-320.

FERNÁNDEZ-PARTAGÁS, J. \& DÍAZ, H. F., 1996 - Atlantic Hurricanes in the second half of the $19^{\text {th }}$ Century. Bulletin of the American Meteorological Society, 77 (12): 28992906.

FERNÁNDEZ TEJEDO, I., 2012 - Fragilidad de un espacio productivo: cambio climático e inundaciones en el Bajío, siglo XVIII. Tzintzun, 55: 107-156.

FERREIRA DA SILVA, G. M., CARAMORI, P. H. \& DA SILVA RICCE, W., 2012 - O jornal como fonte de informação sobre precipitações de granizo no Estado do Paraná. Revista GEONORTE. Edição Especial 2, I (5): 1079-1090.

FLORESCANO, E., 1969 - Precios del maíz y crisis agrícolas en México, 1708-1810: ensayo sobre el movimiento de los precios y sus consecuencias económicas y sociales, 256 рp.; México, D. F.: El Colegio de México, Ediciones Era.

FLORESCANO, E. \& SWAN, S., 1995 - Breve historia de la sequía en México, 246 pp.; México, D. F.: Universidad Veracruzana.

FLORESCANO MAYET, E., SANCHO Y CERVERA, J. \& PÉREZ GAVILÁN ARIAS, D., 1980 Las sequías en México: historia, características y efectos. Comercio exterior, 30 (7): 747-757.

FRANCOU, B., 2004 - Andes del Ecuador: los glaciares en la época de los viajeros (siglos XVIII a XX). In: Los Andes y el reto del espacio mundo. Homenaje a Olivier Dollfus (J.-P. Deler \& É. Mesclier, eds.): 137-152; Lima: Instituto Francés de Estudios Andinos (IFEA), Instituto de Estudios Peruanos (IEP), Embajada de Francia en el Perú.

GARCÍA, E. \& HERNÁNDEZ, M. E., 1988 - Anomalías de la precipitación en la República Mexicana de 1921 a 1980. Revista de Geografía, II (3): 1-24.

GARCÍA, E. \& HERNÁNDEZ, M. E., 1992 - Sequía meteorológica por frecuencia de años muy secos y extremadamente secos. In: Atlas Nacional de México (A. García de Fuentes, ed.), vol. 2: cap. V, núm. 2.9, escala 1:16 000 000; México, D. F.: Instituto de Geografía, Univesidad Nacional Autónoma de México (UNAM).

GARCÍA, E. \& VIDAL, R., 1981 - La tendencia de la precipitación en la parte central de México en los últimos 50 años. Biotica, 6 (1): 103-115. 
GARCÍA, R., DÍAZ, H. F., GARCÍA-HERRERA, R., EISCHEID, J., PRIETO, M. R., HERNÁNDEZ, E., GIMENO, L., RUBIO DURÁN, F. \& BASCARY, A. M., 2001 Atmospheric Circulation Changes in the Tropical Pacific Inferred from the Voyages of the Manila Galleons in the Sixteenth-Eighteenth Centuries. Bulletin of the American Meteorological Society, 82 (11): 2435-2455.

GARCÍA-ACOSTA, V., 1992 - Enfoques teóricos para el estudio histórico de los "desastres naturales". In: Estudios históricos sobre desastres naturales en México (V. GarcíaAcosta, coord.): 19-32; México, D. F.: Centro de Investigaciones y Estudios Superiores en Antropología Social (CIESAS).

GARCÍA-ACOSTA, V., 2006 - Estrategias adaptativas y amenazas climáticas. In: Más allá del cambio climático. Las dimensiones psicosociales del cambio ambiental global (J. Urbina \& J. Martínez, coords.): 29-46; México: Instituto Nacional de Ecología (SEMARNAT), Facultad de Psicología (UNAM).

GARCÍA-ACOSTA, V., PÉREZ ZEVALLOS, J. M. \& MOLINA DEL VILLAR, A., 2003 Desastres Agrícolas en México. Catálogo histórico, Tomo I: Épocas prehispánica y colonial (958-1822), 506 pp.; México, D. F.: Fondo de Cultura Económica (FCE), Centro de Investigaciones y Estudios Superiores en Antropología Social (CIESAS).

GARCÍA HERNÁNDEZ, A., 1997 - Alternativas ante las sequías de 1789-1810 en la Villa de Saltillo, Coahuila, México. In: Historia y Desastres en América Latina, volumen II (V. García-Acosta, coord.): 191-216; Lima: Red de Estudios Sociales en Prevención de Desastres en América Latina (LA RED), Centro de Investigaciones y Estudios Superiores en Antropología Social (CIESAS), Intermediate Technology Development Group (ITDG).

GARCÍA-HERRERA, R., DÍAZ, H. F., GARCÍA, R. R., PRIETO, M. R., BARRIOPEDRO, D., MOYANO, R. \& HERNÁNDEZ, E., 2008 - A Chronology of El Niño Events from Primary Documentary Sources in Northern Peru. Journal of Climate, 21 (9): 19481962.

GARCíA-HERRERA, R., GARCÍA, R. R., PRIETO, M. R., HERNÁNDEZ, E., GIMENO, L. \& DÍAZ, H. F., 2003 - The use of Spanish Historical Archives to reconstruct climate variability. Bulletin of the American Metorological Society, 84 (8): 1025-1035.

GARCÍA-HERRERA, R., RUBiO DURÁN, F., WHEELER, D., HERNÁNDEZ MARTíN, E., PRIETO, M. R. \& GIMENO, L., 2004 - The Use of Spanish and British Documentary Sources in the Investigation of Atlantic Hurricane Incidence in Historical Times. In: Hurricane and Typhoons. Past, Present, and Future (R. J. Murnane \& K.-B. Liu, eds.): 149-176; Nueva York: Columbia University Press.

GARZA MERODIO, G. G., 2002 - Frecuencia y duración de sequías en la cuenca de México de fines del siglo XVI a mediados del XIX. Investigaciones Geográficas, 48: 106-115.

GARZA MERODIO, G. G., 2006 - Climatología histórica: las ciudades mexicanas ante la sequía (siglos XVII al XIX). Investigaciones Geográficas. Boletín del Instituto de Geografía, 63: 77-92.

GASCÓN, M. \& CAVIEDES, C., 2012 - Clima y sociedad en Argentina y Chile durante el periodo colonial. Anuario Colombiano de Historia Social y de la Cultura, 39 (2): 159-185.

GASCÓN, M. \& CAVIEDES, C., 2014 - Etnoclimatología en las pampas: El remonte térmico en la Frontera Sur. Revista TEFROS, 12 (2): 140-154.

GIL-GUIRADO, S., ESPÍN-SÁNCHEZ, J. A. \& PRIETO, M. R., 2016 - Can we learn from the past? Four hundred years of changes in adaptation to floods and droughts. 
Measuring the vulnerability in two Hispanic cities. Climatic Change, 139 (2): 183200.

GIL MONTERO, R., MORALES, M. \& VILLALBA, R., 2010 - Población y economía en los Andes: las crisis de subsistencia en Talina entre los siglos XVII y XX. Surandino Monográfico, 1 (2): 1-33.

GIODA, A. \& PRIETO, M. R., 1999a - Histoire des sécheresses andines. Potosí, El Niño et le Petit Âge Glaciaire. La Météorologie. Revue de la Société Météorologique de France, 8 (27): 33-42.

GIODA, A. \& PRIETO, M. R., 1999b - Para una historia climática de La Paz en los últimos cinco siglos. Historias de la ciudad de La Paz. Bolivia. Revista de la coordinadora de Historia, 3: 13-33.

GUERRIDO, C., VILLALBA, R. \& ROJAS, F., 2014 - Documentary and tree-ring evidence for a long-term interval without ice impoundments from Glaciar Perito Moreno, Patagonia, Argentina. The Holocene, 24 (12): 1686-1693.

HAMILTON, K. \& GARCÍA, R. R., 1986 - El Niño/Southern Oscillation Events and Their Associated Midlatitude Teleconnections 1531-1841. Bulletin of the American Meteorological Society, 67 (11): 1354-1361.

HERRERA, R. G., PRIETO, M. R. \& ROJAS, F., 2011 - Lluvias, sequías e inundaciones en el Chaco semiárido argentino entre 1580 y 1900. Revista de la Junta de Estudios Históricos de Santa Fe, LXIX: 173-200.

HUERTAS VALLEJOS, L., 1993 - Anomalías cíclicas de la naturaleza y su impacto en la sociedad: "El fenómeno El Niño". Bulletin de I'Institut Français d'Études Andines, 22 (1): 345-393.

HUERTAS VALLEJOS, L., 2001 - Diluvios Andinos a través de las fuentes documentales, 392 pp.; Lima: Fondo Editorial de la Pontificia Universidad Católica del Perú.

JÁUREGUI, E., 1979 - Algunos aspectos de las fluctuaciones pluviométricas en México en los últimos cien años. Boletín del Instituto de Geografía, 9: 39-64.

LE ROY LADURIE, E., 1990 [1967] - Histoire du climat depuis I'an mil, 287 pp.; París: Flammarion.

LIVERMAN, D. M., 1990 - Drought impacts in Mexico: climate, agriculture, technology, and land tenure in Sonora and Puebla. Annals of the Association of American Geographers, 80 (1): 49-72.

LÓPEZ DE ALBORNOZ, C., 1997 - Crisis agrícolas y crisis biológicas en la jurisdicción de San Miguel de Tucumán durante la segunda mitad del siglo XVIII. In: Historia y Desastres en América Latina, volumen II (V. García-Acosta, coord.): 163-190; Lima: Red de Estudios Sociales en Prevención de Desastres en América Latina (LA RED), Centro de Investigaciones y Estudios Superiores en Antropología Social (CIESAS), Intermediate Technology Development Group (ITDG).

MARKGRAF, V., 2000 - Interhemispheric Climate Linkages, XXI + 454 pp.; California: Academic Press.

MARTíNEZ CHAVES, P. A., BETANCOURT MENDIETA, A., CARETTA, M. N. \& AGUILAR ROBLEDO, M., 2010 - Procesos históricos y ambientales en Cerro de San Pedro, San Luis Potosí, México, 1948-1997. Región y Sociedad, XXII (48): 211-241.

MASIOKAS, M. H., VILLALBA, R., CHRISTIE, D. A., BETMAN, E., LUCKMAN, B. H., LE QUESNE, C., PRIETO, M. R. \& MAUGET, S., 2012 - Snowpack variations since AD 1150 in the Andes of Chile and Argentina $\left(30^{\circ}-37^{\circ} \mathrm{S}\right)$ inferred from rainfall, tree-ring and documentary records. Journal of Geophysical Research: Atmospheres, 117 (D5): 1-11. 
MENDOZA, B., GARCÍA-ACOSTA, V., VELASCO, V., JÁUREGUI, E. \& DÍAZ-SANDOVAL, R., 2007 - Frequency and duration of historical droughts from the 16th to the 19th centuries in the Mexican Maya lands, Yucatan Peninsula. Climatic Change, 83 (1-2): 151-168.

MENDOZA, B., JÁUREGUI, E., DIAZ-SANDOVAL, R. , GARCÍA-ACOSTA, V., VELASCO, V. \& CORDERO, G., 2005 - Historical Droughts in Central Mexico and Their Relation with El Niño. Journal of Applied Meteorology, 44 (5): 709-716.

METCALFE, S. E., 1987 - Historical data and climatic change in Mexico-A review. The Geographical Journal, 153 (2): 211-222.

METCALFE, S. E., SAY, A., BLACK, S., McCULLOCH, R. \& O'HARA, S. L., 2002 - Wet Conditions during the Last Glaciation in the Chihuahuan Desert, Alta Babicora Basin, Mexico. Quaternary Research, 57 (1): 91-101.

MORA PACHECHO, K. G., 2015 - Los agricultores y ganaderos de la sabana de Bogotá frente a las fluctuaciones climáticas del siglo XVIII. Fronteras de la Historia, 20 (1): 14-42.

MUSSET, A., 1992 - El agua en el Valle de México: siglos XVI-XVIII, 245 pp.; México, D. F.: Pórtico de la Ciudad de México, Centro de Estudios Mexicanos y Centroamericanos (CEMCA).

NEUKOM, R., PRIETO, M. R., MOYANO, R., LUTERBACHER, J., PFISTER, C., VILLALBA, R., JONES, P. D. \& WANNER, H., 2009 - An extended network of documentary data from South America and its potential for quantitative precipitation reconstructions back to the 16th century. Geophysical Research Letters, 36 (L12703): 1-5.

O'HARA, S. L. \& METCALFE, S. E., 1995 - Reconstructing the climate of Mexico from historical records. The Holocene, 5 (4): 485-490.

ORTLIEB, L., 1994 - Las mayores precipitaciones históricas en Chile central y la cronología de eventos ENOS en los siglos XVI-XIX. Revista Chilena de Historia Natural, 67: 463-485.

ORTLIEB, L., 1995 - Eventos El Niño y episodios lluviosos en el desierto de Atacama: el registro de los dos últimos siglos. Bulletin de I'Institut Français d'Études Andines, 24 (3): 519-537.

ORTLIEB, L., 2000 - The Documented Historical Record of El Niño Events in Peru: An Update of the Quinn Record (Sixteenth through Nineteenth Centuries). In: El Niño and the Southern Oscillation: Multiscale Variability and Global and Regional Impacts (H. F. Díaz \& V. Markgraf, eds.): 207-295; Cambridge, Nueva York: Cambridge University Press.

ORTLIEB, L., 2013 - Simposio Internacional «15 000 años de clima y hombre en los Andes centrales. Enfoques paleoclimatológicos y arqueológicos». Lima, 22-24 de agosto de 2013. Bulletin del Institut Français d'Études Andines, 42 (3): 552-555.

PABÓN CAICEDO, J. D., 2006 - El clima de Colombia durante los siglos XVI-XIX a partir de material histórico. Parte I: Inventario de fuentes de información. Cuadernos de Geografía: Revista Colombiana de Geografía, 15: 75-92.

PADILLA LOZOYA, R., 2016 - El surgimiento de una sociedad vulnerable y sus respuestas ante amenazas naturales: San José del Cabo, Baja California Sur, siglos XVI al XVIII. In: Clima, desastres y convulsiones sociales en España e Hispanoamérica, siglos XVII-XX (L. A. Arrioja Díaz Virruell \& A. Alberola Romá, eds.): 243-268; Alicante: Universidad de Alicante, El Colegio de Michoacán.

PALACIOS, G., 1996 - La agricultura campesina en el nordeste oriental del Brasil y las sequías de finales de siglo XVIII. In: Historia y Desastres en América Latina, volumen I (V. García-Acosta, coord.): 221-258; Santafe de Bogotá: Red de Estudios 
Sociales en Prevención de Desastres en América Latina (LA RED), Centro de Investigaciones y Estudios Superiores en Antropología Social (CIESAS).

PENTIMALLI DE NAVARRO, M. \& RODRÍGUEZ OSTRIA, G., 1988 - Las razones de la multitud (hambrunas, motines y subsistencia: 1878-79). Estado y Sociedad: Revista boliviana de Ciencias Sociales, 4 (5): 15-33.

PFISTER, C., BRÁZDIL, R., OBREBSKA-STARKEL, B., STARKEL, L., HEINO, R. \& VON STORCH, H., 2001 - Strides Made in Reconstructing Past Weather and Climate. EOS, Transactions. American Geophysical Union, 82 (22): 248.

POLITIS, G. G., 1984 - Climatic variations during historical times in Eastern Buenos Aires Pampas, Argentina. In: Quaternary of South America and Antarctic Peninsula, volumen 2 (J. Rabasa, ed.): 133-161; Rotterdam.

POVEDA, G. \& MESA, O. J., 1996 - Las fases extremas del fenómeno ENSO (El Niño y La Niña) y su influencia sobre la hidrología de Colombia. Ingeniería Hidráulica en México, XI (1): 21-37.

PRIETO, M. R., 1981 - Historia del clima de la región cuyana. Aproximación metodológica. Cuadernos del CEIFAR, 6: 115-128.

PRIETO, M. R., 1983 - El clima de Mendoza durante los siglos XVII y XVIII. Meteorológica, XIV (1-2): 165-174.

PRIETO, M. R., 1985 - Relación entre clima, condiciones ambientales y asentamientos humanos en la provincia de Mendoza en los siglos XVI, XVII y XVIII. Revista de Historia de América, 100: 79-118.

PRIETO, M. R., 1986 - The glacier dam on the Rio Plomo: a cyclic phenomenon? Zeitschrift fur Gletscherkunde und Glazialgeologie, 22 (H. 1): 73-78.

PRIETO, M. R., 2007 - Enso Signals in South America: Rains and Floods in the Parana River during Colonial Times. Climatic Change, 83 (1-2): 39-54.

PRIETO, M. R., 2009 - The Floods of the Paraná River during the Spanish Colonial Period: Impacts and Responses. In: Natural Disasters, Cultural Responses: Case Studies toward a Global Environmental History (C. Mauch \& C. Pfister, eds.): 285-303; Lanham: Lexington Books.

PRIETO, M. R., 2011 - Las inundaciones en el río Paraná durante el período colonial. Impacto socio-cultural y respuestas adaptativas. Revista de la Junta Provincial de Estudios Históricos de Santa Fe, LXVIII: 15-44.

PRIETO, M. R., GALLEGO, D., GARCÍA-HERRERA, R. \& CALVO, N., 2005 - Deriving Wind Force Terms from Nautical Reports Through Content Analysis. The Spanish and French cases. Climatic Change, 73 (1-2): 37-55.

PRIETO, M. R. \& GARCÍA-HERRERA, R., 2009 - Documentary sources from South America: Potential for climate reconstruction. Palaeogeography, Palaeoclimatology, Palaeoecology, 281 (3-4): 196-209.

PRIETO, M. R., GARCÍA-HERRERA, R. \& HERNÁNDEZ MARTIN, E., 2004 - Early Records of Icebergs in the South Atlantic Ocean from Spanish Documentary Sources. Climatic Change, 66 (1-2): 29-48.

PRIETO, M. R. \& HERRERA, R. G., 1998 - Naos, clima y glaciares en el Estrecho de Magallanes durante el siglo XVI. Anuario de Estudios Americanos, LV (2): 413-439.

PRIETO, M. R. \& HERRERA, R. G., 2002 - Clima y economía en el área surandina: El Alto Perú y el espacio económico regional a fines del siglo XVIII. In: Estudios sobre historia y ambiente en América, II: Norteamérica, Sudamérica y el Pacífico (B. García Martínez \& M. R. Prieto, comps.): 55-80; México, D. F.: El Colegio de México, Instituto Panamericano de Geografía e Historia (IPGH). 
PRIETO, M. R., HERRERA, R. G., CASTRILLEJO, T. N. \& DUSSEL, P., 2001 - Variaciones climáticas recientes y disponibilidad hídrica en los Andes Centrales Argentinochilenos (1885-1996). El uso de datos periodísticos para la reconstitución del clima. Meteorológica, 25 (1-2): 27-43.

PRIETO, M. R., HERRERA, R. G. \& DUSSEL, P., 1999 - Historical Evidences of Streamflow Fluctuations in the Mendoza River, Argentina, and their relationship with ENSO. The Holocene, 9 (4): 473-481.

PRIETO, M. R., HERRERA, R. G. \& DUSSEL, P., 2000 - Archival Evidence for some Aspects of Historical Climate Variability in Argentina and Bolivia during the 17th and 18th Centuries. In: Southern Hemisphere Paleo- and Neoclimates (P. Smolka \& W. Volkheimer, eds.): 127-142; Berlín-Heidelberg: Springer-Verlag.

PRIETO, M. R. \& RICHARD JORBA, R., 1991 - Anomalías climáticas en la Cuenca del Plata y el NOA y sus consecuencias socioeconómicas. Siglos XVI, XVII y XVIII. Leguas. Revista Argentina de Geografía, 1: 41-104.

PRIETO, M. R. \& ROJAS, F., 2012 - Documentary evidence for changing climatic and anthropogenic influences on the Bermejo Wetland in Mendoza, Argentina, during the 16th-20th century. Climate of the Past, 8: 951-961.

PRIETO, M. R. \& ROJAS, F., 2015 - Determination of droughts and high floods of the Bermejo River (Argentina) based on documentary evidence (17th to 20th century). Journal of Hydrology, 529 (Part 2): 676-683.

PRIETO, M. R., SOLARI, M. E., CROUCHET, J. \& LARROUCAU, A., 2012 - Fuentes documentales para el estudio del clima en la región sur-austral de Chile $\left(40^{\circ}-51^{\circ} \mathrm{S}\right)$ durante los últimos siglos. Bosque, 33 (2): 135-144.

PRIETO, M. R., SORIA, D. \& HERRERA, R. G., 1995 - Granizo, heladas y vitivinicultura en Mendoza (1887-1987). Revista de Estudios Regionales, Centro Interdisciplinario de Estudios Regionales (CEIDER), 13-14: 151-185.

QUINN, W., 1992 - A Study of Southern Oscillation-Related Climatic Activity for AD 6221900 Incorporating Nile River Flood Data. In: El Niño: Historical and Paleoclimate Aspects of the Southern Oscillation (H. F. Díaz \& V. Markgraf, eds.): 119-149; Cambridge, Nueva York: Cambridge University Press.

QUINN, W. H. \& NEAL, V. T., 1983 - Long-term variations in the Southern Oscillation, El Niño, and Chilean subtropical rainfall. Fishery Bulletin, 81 (2): 363-374.

QUINN, W. H., NEAL, V. T. \& ANTÚNEZ DE MAYOLO, S. E., 1987 - El Niño Occurrences over the Past Four and a Half Centuries. Journal of Geophysical Research, 92 (C13): 14449-14461.

QUINN, W. H., ZOPF, D. O., SHORT, K. S. \& KUO YANG, R. T. W., 1978 - Historical trends and statistics of the Southern Oscillation, El Nino, and Indonesian droughts. Fishery Bulletin, 76 (3): 663-678.

RAPPAPORT, E. N. \& FERNÁNDEZ-PARTAGÁS, J. J., 1997 - History of the deadliest Atlantic tropical cyclones since the discovery of the New World. In: Hurricanes, Climate and Socioeconomic Impacts (H. F. Díaz \& R. S. Pulwarty, eds.): 93-108; Berlín: SpringerVerlag.

RHOADES, P., 2008 - Disappearance of the glacier on Mama Cotacachi: Ethnoecological research and climate change in the Ecuadorian Andes. Pirineos, 163: 37-50.

RICHARDSON GILL, B., 2001 - The Great Maya Droughts: Water, Life, and Death, xx + 464 pp.; Alburquerque: University of New Mexico Press.

SÁNCHEZ-CORTÉS, M. S. \& LAZOS CHAVERO, E., 2011 - Indigenous perception of changes in climate variability and its relationship with agriculture in a Zoque community of Chiapas, Mexico. Climate Change, 107 (3-4): 363-389. 
SCHLÜPMANN, J., 2003 - Dîme, production agricole et événements climatiques : le cas de Piura au nord du Pérou (17-19ème siècles). Bulletin de l'Institut Français d'Études Andines, 32 (2): 263-292.

TANDETER, E. \& WACHTEL, N., 1984 - Precios y producción agraria. Potosí y Charcas en el siglo XVIII, 95 pp.; Buenos Aires: Centro de Estudios de Estado y Sociedad (CEDES).

TASSO, A., 2011 - La sequía de 1937 en Santiago del Estero. Antecedentes y consecuencias de un acontecimiento ambiental. Trabajo y Sociedad, XV (17): 17-39.

TAULIS, E., 1934 - De la distribution des pluies au Chili. Matériaux pour l'étude des calamités. Société de Géographie de Genève, Parte 1: 3-20.

TERNEUS, A. \& GIODA, A., 2006 - In search of colonial El Nino events and a brief history of meteorology in Ecuador. Advances in Geosciences, 6: 181-187.

TORTOLERO, A., 1996 - Historia Agraria y medio ambiente en México: Estado de la Cuestión. Historia Agraria. Revista de agricultura e historia rural, 11: 151-178.

URRUTIA DE HAZBÚN, R. \& LANZA LAZCANO, C., 1993 - Catástrofes en Chile, 1541 1992, 440 pp.; Santiago de Chile: Editorial La Noria.

VICUÑA MACKENNA, B., 1970 - El Clima de Chile: ensayo histórico, 399 pp.; Buenos Aires: Editorial Francisco de Aguirre.

VIDAL, R. \& GARCÍA, E., 1980 - The trend of precipitation in several regions of Mexico. Main Section. Memoria del XXIV Congreso Internacional de Geografía: 114-195.

VILLA, M. A., 2000 - Vida e morte no sertão: história das secas no Nordeste nos séculos XIX e XX, 269 pp.; São Paulo: Ática.

VILLALBA, R., 1994 - Tree-ring and glacial evidence for the Medieval Warm Epoch and the Little Ice Age in Southern South America. Climatic Change, 26: 183-197.

WALSH, R. P. D. \& READING, A. J., 1991 - Historical changes in tropical cyclone frequency within the Caribbean since 1500. Würzburger Geographische Arbeiten, 80: 199240.

WANNER, H., BEER, J., BÜTIKOFER, J., CROWLEY, T. J., CUBASCH, U., FLÜCKIGER, J., GOOSSE, H., GROSJEAN, M., JOOS, F., KAPLAN, J. O., KÜTTEL, M., MÜLLER, S. A., PRENTICE, I. C., SOLOMINA, O., STOCKER, T. F., TARASOV, P., WAGNER, M. \& WIDMANN, M., 2008 - Mid- to Late Holocene climate change: an overview. Quaternary Science Reviews, 27 (19-20): 1791-1828. 